\title{
Meta
}

Journal des traducteurs

Translators' Journal

\section{La traduction en anglais de la littérature francophone : perception du phénomène au Nigéria}

\section{Bassey E. Antia}

Volume 44, numéro 3, septembre 1999

URI : https://id.erudit.org/iderudit/001893ar

DOI : https://doi.org/10.7202/001893ar

Aller au sommaire du numéro

Éditeur(s)

Les Presses de l'Université de Montréal

ISSN

0026-0452 (imprimé)

1492-1421 (numérique)

Découvrir la revue

Citer cet article

Antia, B. E. (1999). La traduction en anglais de la littérature francophone : perception du phénomène au Nigéria. Meta, 44(3), 517-521.

https://doi.org/10.7202/001893ar
Résumé de l'article

Cet article présente un bref historique du phénomène de la traduction vers l'anglais de la littérature africaine de langue française. On y aborde la fonctionnalité de la traduction au Nigeria : la découverte de l'Autre; puis l'auteur fait un survol critique de certaines de ces traductions. Elle conclut que ce type de traduction aurait avantage à être éditées en Afrique. 


\section{BLOC-NOTES}

\section{La traduction en anglais de la littérature francophone: perception du phénomène au Nigeria*:}

\begin{abstract}
RÉSUMÉ
Cet article présente un bref historique du phénomène de la traduction vers l'anglais de la littérature africaine de langue française. On y aborde la fonctionnalité de la traduction au Nigeria: la découverte de l'Autre; puis l'auteur fait un survol critique de certaines de ces traductions. Elle conclut que ce type de traduction aurait avantage à être éditées en Afrique.
\end{abstract}

\section{ABSTRACT}

This article gives a brief historical perspective on the translation of French African literature into English. It looks at the role of translation in Nigeria-discovering the Other-and goes on to provide a critical overview of some translated works. It concludes that this type of translation should be edited in Africa.

Lidée qu'on se fait de la traduction varie d'un contexte à un autre ou d'un cadre spatio-temporel à un autre. En ce qui concerne l'Occident, deux visions de la traduction se dégagent de l'époque où l'on tenait des propos philosophiques sur le langage. La traduction fut considérée soit comme traduction, opération légitime et utile; soit comme traducianisme, notion péjorative, voire hérétique, faisant corps avec l'épigramme italienne traduttore, traditore. Cette dernière vue est sans doute périmée aujourd'hui.

Au Nigeria, la perception du phénomène est différente. S'il est vrai qu'on a toujours eu conscience de la traduction orale (l'interprétariat) parce que pratiquée dans les églises et les tribunaux, il reste que sa contrepartie écrite est restée ignorée pendant longtemps. En effet, en dehors des universitaires spécialistes de la littérature, peu se rendent compte que certains textes qu'ils lisent ne sont pas des créations originales, mais des traductions. C'est déjà là une première perception (ou mieux encore: une non-perception) du phénomène de la traduction en général. Mais limitonsnous à ceux qui en ont conscience.

Il nous semble qu'au Nigeria, la traduction en anglais de la littérature africaine francophone est perçue de deux façons. Il y a d'une part ceux qui y voient une ouverture sur l'extérieur - c'est là la vision fonctionnelle - d'autre part, il existe une optique critique selon laquelle la traduction est envisagée dans sa valeur intrinsèque: la traduction qua traduction. Le phénomène des traductions réalisées par les étudiants d'université reste encore à étudier.

Avant de développer les deux visions retenues pour la discussion, esquissons au préalable l'historique du phénomène de la traduction en anglais de la littérature africaine d'expression française.

\section{GENÈSE ET DÉVELOPPEMENT DU PHÉNOMÈNE DE LA TRADUCTION EN ANGLAIS DE LA LITTÉRATURE AFRICAINE FRANCOPHONE}

Ce phénomène aurait commencé en 1955 avec la publication de The $D$ ark Child, la première traduction anglaise de L'enfant noir, un roman de Camara Laye. En 1959, la maison d'édition Fontana a publié la traduction de James Kirkup, The African Child, qui connut plus de succès que la précédente. Dès lors, les grands noms de la littérature africaine d'expression française _ de Senghor et Beti à F. Oyono en passant par Yambo Ouologuem et Cheikh Hamidou Kane pour arriver à Mariama Bâ _ ont été traduits ${ }^{1}$.

On constate, dans l'index de Timothy-Asobele, qu'entre 1962 et 1970, des traductions sont publiées à la cadence de deux titres par an, à l'exception de 1963 et 1966 qui enregistrent chacune une seule traduction (1960 et 1961 n'enregistrent rien). L'année 1971 marque un sommet: elle compte, à elle seule, cinq traductions éditées. Depuis cette date jusqu'en 1984, quand s'arrête la bibliographie, la plupart des années n'ont vu paraitre qu'une seule traduction.

On constate également que, mis à part la traduction réalisée par la Nigériane Modupe Johnson (So Long a Letter), toutes les autres qui figurent dans l'index ont été réalisées par des Occidentaux: James Kirkup, Gerald Moore, J. Reed, Clive Wake, Peter Green, P. J. Oswald, Dorothy Blair, J. D. Pickett, Ralph Manheim, pour ne mentionner que ceux-là.

Ce sont les maisons d'édition comme Heinemann (Londres et Ibadan) qui rendent possible ce phénomène. Heinemann de Londres a publié 11 des 32 titres de l'index et cela de 1962 à 1971. Heinemann d'Ibadan aurait commencé l'édition de traductions en 1971. Cette succursale a publié huit traductions. À la seule exception de The Death of Chaka, paru chez Oxford University Press à Nairobi, les autres titres de l'index sont publiés dans des métropoles occidentales. 


\section{LA PERCEPTION FONCTIONNELLE}

La perception fonctionnelle de la traduction se décèle surtout dans le champ de la littérature comparée dont le but principal nous semble être «la découverte de l'Autre». C'est autour de cet objectif que s'articulent les réflexions qui suivent.

À l'école, mais aussi à l'université, les cours de littérature sont axés aussi bien sur les écrivains anglophones que sur les auteurs francophones. Ces derniers sont évidemment étudiés en traduction anglaise. À titre d'exemple, on peut remarquer que Birago Diop, Ferdinand Oyono, Sembène Ousmane - écrivains francophones - figurent au programme de littérature des écoles secondaires au Nigeria. De la même manière, The Ambiguous Adventure, The Old Man and the Medal, The Poor Christ of Bomba - toutes des traductions - sont étudiés dans les départements d'anglais des universités nigérianes. C'est grâce à la traduction que les étudiants nigérians peuvent voir des parallèles et des asymétries thématiques entre la littérature du Commonwealth d'Afrique et celle de la Francophonie africaine.

Souvent, la découverte qui en résulte fournit souvent matière à une nostalgie ou à un romantisme africains. Le Nigerian qui lit en anglais Les contes d'Ahmadou Koumba issus de la tradition orale wolof du Sénégal et qui y trouve des parallèles dans sa tradition efik, edo ou nupe se réjouit de sa découverte. Dans un élan nostalgique, il regagne l'Afrique sans frontières des années précoloniales et regrette, d'emblée, la balkanisation de son continent. Il se rend compte de la singularité du patrimoine culturel africain.

La possibilité qu'ont les Nigérians de dialoguer avec la Francophonie provient de la traduction. La valeur de ce contact est inestimable. Dans son article, où elle étudie la perception critique de l'œuvre de Camara Laye, Adele King (19??) affirme que:

Les traductions en anglais de l'œuvre de Laye ont servi à établir un dialogue critique entre les Anglophones et les Francophones d'Afrique de l'Ouest; en effet, la littérature critique sur Laye provient moins des Francophones que des Anglophones. (Notre traduction)

En d'autres termes, sans la traduction, on n'aurait pas eu toute cette masse de métalittérature critique sur Laye.

C'est également ce genre de contact avec les idées en cours de l'autre côté du rideau linguistique ouest-africain qui a donné naissance à ce qu'on pourrait à raison considérer comme la polémique la plus fameuse en lettres africaines: la Négritude contre la Tigritude, qui a opposé Senghor à Soyinka. Ce dernier, déjà célèbre, critiquera les prophètes de la négritude, déclarant que le céphalophe devrait se faire valoir moins par un narcissisme outrancier que par ses bonds ${ }^{2}$. Et, à la conférence des écrivains africains tenue à Kampala en 1962, Soyinka restera fidèle à sa conviction: le tigre ne proclame pas sa tigritude. Ce qui est intéressant dans tout cela, c'est que Soyinka n'avait pas de compétence en français au moment où il s'opposait à Senghor. Il s'ensuit que si Soyinka n'avait pas eu la possibilité de lire Les hosties noires de Senghor en traduction anglaise, ce débat n'aurait probablement pas eu lieu.

Le caractère fonctionnel de la traduction au Nigeria se voit aussi dans l'attribution des prix littéraires aux écrivains francophones. En 1987, le prix Okigbo de poésie a été décerné à un poète d'expression française, Jean Baptiste Tati-Loutard. En choisissant Tati-Loutard qui lui est accessible en traduction anglaise, le jury de l'Association des écrivains nigérians (Association of $\mathrm{N}$ igerian Authors - ANA) semble faire deux choses simultanément: couronner un écrivain prodigieux et faire l'éloge de la traduction.

\section{L'OPTIQUE CRITIQUE}

Deux études, celle d'Adele King (19??) portant sur Kirkup et la nôtre (Antia 1988) sur Manheim, reflètent cette perspective critique de la traduction. Les réflexions dans ces études semblent s'organiser autour de deux pôles. D'une part, il y a des questions langagières et d'isomorphisme textuel et d'autre part, des problèmes idéologiques.

Sur le plan de la textualité, on identifie plusieurs genres d'écarts. Commençons par l'omission. La description que fait Laye du pouvoir magique de sa mère ${ }^{3}$ manque dans la traduction de Kirkup (The African Child). De même, une partie des propos philosophiques tenus par le père Laye sur le destin scolaire de son fils, de ses oncles et de lui-même ${ }^{4}$ n'apparaît pas dans la traduction. Est également omis l'interdit selon lequel les circoncis ne devaient pas être vus nus pas des femmes ${ }^{5}$.

Le phénomène d'omission, nous le constatons aussi dans la traduction anglaise de Le devoir de violence (1968: 133) réalisée par Ralph Manheim (1971: 155). Tous le sentiment affiché par Kadidia à la réunion avec son frère dans un bordel parisien $^{6}$ est perdu dans la traduction. Observons un autre exemple de non-isomorphisme entre le texte français et la traduction anglaise dans Bound to Violence. les phrases «Kratonga visa de nouveau»/«Again Kratonga took an aim» et "C'était un juste»/«He was a righteous man » encadrent des comptes rendus bien différents de l'humiliation de Vandame, le gouverneur, par Wampoulo et Kratonga ${ }^{7}$.

Toujours dans cette optique textuelle/ langagière, King et Antia relèvent des exemples de bévues sémantiques et grammaticales chez les tra- 
ducteurs étudiés. Kirkup (1959: 133) traduit «contrôler ce que j'avais fait» (Laye 1966: 149) par "criticise what I had done» plutôt que par check. Manheim (1971: 5) traduit «l'année en cours» (Ouologuem 1968: 11) par «during the coming year » plutôt que par «in the course of theyear ». " Je me doutais bien" (Laye 1966: 158) devient chez Kirkup (1959: 141) «I did not suspect» alors que pour «il demeura ainsi, sans rien dire, le regard mélancolique» et "les accusations [...] d'avoir engrossé trois négresses» (Ouologuem 1968: 50 et 92) Manheim (1971: 37 et 38) - ou est-ce une erreur d'impression? - produit les non-phrases "so he remained, silent and melancholy» et "the accusation [...] that he got three black women with child». Il faut, à la première, la forme adjectivale «melancholic» et, à la deuxième, le mot «heavy».

Et la simple phrase:

Tour à tour redoutable, il défit les Berbères, les Maures et les Touaregs, reconnut le Cheik Adberrahman Es Soyouti, secourut le Cheik Mohammed ben Abdelkarim El-Meghili. (Ouologuem 1968: 13)

produit chez Manheim la confusion qu'est:

Terrible in battle, he defeated the Berbers, the Moor and the Tuareg, recognized the Sheikh M ohammed ben Abd-al-Karim al-M eghili. (Manheim 1971: 7)

Le Saif a reconnu le Cheik Es Soyouti et non le Cheik El-Meghili. Aucune mention n'est faite du Cheik Es Soyouti en traduction. Le Cheik ElMeghili a été secouru et non reconnu.

La valorisation de la vision du monde d'où est issu le traducteur constitue l'angle idéologique de la perspective critique. Très subtile, elle se traduit de plusieurs façons.

Commençons par l'insensibilité à la «manière africaine de dire », c'est-à-dire l'ignorance ou le mépris des conventions linguistiques africaines. Le principe de fréquence équivalente au sens développé par Newmark (1982: 145) nous apprend qu'un mot ou une image peut avoir un équivalent en langue cible sans pour autant que cet équivalent soit juste parce que peu usité ou incompatible avec les habitudes ou les pratiques sociales des récepteurs de la traduction. Il en est souvent question lorsque l'écrivain et son traducteur sont de cultures différentes.

King fait remarquer que Kirkup emploie une métaphore étrange dans le contexte guinéen de L'enfant noir lorsqu'il traduit «tout rebondi» (p. 50) par «plump as patridge» (p. 37). Manheim rend «désireux d'apaiser les mânes de son père mort» (p. 16) par «wishing to appease the shades of his dead father» (p. 10). Dans cette expression du cosmos mystique africain, le mot «spirits» s'associe mieux avec les morts et les ancêtres. De même «trail» (p. 28) et «rooster» (p. 98), équivalents proposés par Manheim pour «piste» (p. 36) et «coq" (p. 114) ont une fréquence inférieure à celle de «footpath» et à «cock».

Il revient également à servir un intérêt autre que celui des lecteurs africains que de rendre «l'arbre à palabres» (Ouologuem 1968: 23) pas «council tree» (p. 17) parce que le mot "palavar» (comme dans «palavar tree»), normal dans l'anglais parlé en Afrique, risquerait de dérouter les lecteurs occidentaux. C'est comme si l'on rendait la «case à palabres» par "council chambers». Et ce n'est qu'un traducteur ouvert à la stylistique africaine qui aurait su servir de "complément» à Ouologuem en traduisant "Nommé donc empereur» (1968: 24) non pas par "Appointed emperor» comme le fait Manheim (1971: 17), mais par une métonymie-insigne de la royauté: turbaned, crowned, elected (selon les circonstances). Turbaned convient mieux au contexte musulman du texte.

Le réalisme linguistique dont il est question se manifeste aussi sur le plan des niveaux de langue. Plus que dans l'Occident, la différenciation des classes sociales sur la base du critère scolaire est très importante en Afrique. C'est dire que les écarts de la norme grammaticale dans un texte africain constituent à peine de libres variations. Manheim ignore l'expressivité de ces écarts, comme le révèle les extraits suivants:

1. Pourquoi qu'tu n'vas point che les Flencessi avec ton bon nègre blanc au lieu de feignanter par itou? (155):

Why don't you go to the Flencessi with your white nigger instead of hanging around here? (134)

2. T'en as de la veine (164): You're lucky (143)

C'est pas des menteries au moins? (164): You're not pulling my legs? (143)

3. T'en as connu des étudiants originaires de Tillaberi-Bentia, par hasard? (1964):

Ever met any students from Tillaberi-Bentia, by any chance? (1943)

4. Mais un type donc, quelqu'un quelqu'un comme moi, une femme quoi (1964):

oh, just a guy, somebody like me. Well a woman. (1943)

Les phrases du français «petit-nègre» renseignent sur les énonciateurs, leur niveau de scolarité et, de là, sur leur logique. Alors que la première est prononcée par les villageois illettrés, les trois autres sont prononcées par Kadidia, un raté scolaire. Cette valeur sémantique supplémentaire est perdue en traduction. Le traducteur aurait pu se b a $\quad s \quad$ e $\quad r$ sur l'anglais pidgin, ce qui donnerait, par exemple: 
1) Why you no go di French people dem country instead of to remain here; 2) You get luck; 3) $\mathrm{Na}$ true?; 4) You sabi any student wey come from Tillaberi-Bentia; 5) Well, na person, person like me. Okay, woman.

La valorisation de la perspective du traducteur plutôt que celle du texte ne se trahit pas seulement par cette insensibilité à la "manière africaine de dire». Elle est révélée aussi par des sous-entendus idéologiques.

Si ces derniers propos résultent d'une ignorance chez le traducteur, on ne saurait pas dire la même chose pour certains ajouts à résonance idéologique. Laye, comme le fait remarquer King, se rend compte qu'il écrit pour des lecteurs étrangers, d'où la qualification de plusieurs actions et événements par la locution prépositionnelle «chez nous ", "our country» (p. 29 et p. 15). Cependant, la distance que témoigne le traducteur envers son texte, The African Child, une distance trahie par l'ajout du pronom possesif dans «our December» (p. 46) pour le simple « décembre» (p. 59) ou celui, dans $A$ D ream of Africa, de l'adverbe "there» dans «school teachers are still on holiday there in the month of September ", cette distance donc prête au soupçon. King attire également l'attention sur la traduction de «simple plaisir» par «primitive delight» et de «la danse du pays» comme "native dance» (The Radiance of the King).

Une traduction qui, à notre avis, prête également au soupçons idéologique est celle de «aspect sombre» (Laye 1966: 80) «black side» (Kirkup 1959: 68) et de "côté sombre» (p. 81) par «black side» (p. 68). C’est Laye qui raconte le côté désagréable de sa vie d'écolier. En dépit de l'institutionnalisation dans le vocabulaire de l'anglais des stéréotypes raciaux, l'emploi de black avec une connotation négative et dans un texte où est valorisé, par un mouvement nostalgique, tout ce qui est associé à cette couleur est sans doute à revoir. Surtout lorsque la deuxième occurrence de ce mot est mise en relief par des guillemets. C'est faire prendre à un piège linguistique inutile le petit Laye, susceptible, de ce fait, de devenir un objet de risée dans une analyse anti-négritude.

Comme Kirkup, Manheim (1971) exagère parfois, rendant pertinente dans sa traduction la question de la dimension idéologique même si le problème se pose à peine ici, la perspective de Yambo Ouologuem correspondant à la vision occidentale de l'Afrique (voir Lamb 1985). Il traduit «moricauds» (Ouologuem 1968: 154) qu'il avait déjà rendu par «jigaboos» (p. 66), par «black savages» (p. 133). De par cet équivalent, qui n'était pas hors portée pour Ouologuem dans Ledevoir de violence, le passage anglais prend davantage un timbre péjoratif.

Cette perception critique du phénomène de la traduction au Nigeria n'est pas, comme le laisse- rait croire ce qui précède, seulement négative. Par rapport à la totalité des textes d'où elles sont tirées, le pourcentage de ces critiques négatives ne saurait dépasser celui des points positifs. Nous avons, par exemple, loué Ralph Manheim ailleurs (Antia 1988) pour l'économie des moyens, l'explicitation de certaines idées implicites dans l'original, sa sensibilité aux nuances de multiples aspects et pour le remaniement de la syntaxe de Yambo Ouologuem, facilitant, ce faisant, la lecture pour l'esprit anglophone qui est moins abstrait.

\section{CONCLUSION}

Nous avons donc deux perceptions du phénomène de la traduction en anglais de la littérature africaine francophone. Perçue fonctionnellement, elle est au service de la littérature et cela de maintes façons. Envisagées à partir d'un point de vue critique, elle enrichit la traductologie et profite à l'industrie éditoriale.

Mais au-delà de ces simples constatations, cette étude a révélé le pourquoi et le comment de «l'expatriation» des œuvres littéraires africaines. Devant ces faits, un défi s'impose, celui de «rapatrier» ces œuvres au moyen des traductions réalisées et éditées en Afrique pour un public africain.

Bassey Antia

Université de Maiduguri, Maiduguri, Nigeria

\section{NOTES}

* Nous exprimons notre gratitude à M. TimothyAsobele dont l'apport à la conception de cet article est inestimable.

1. Timothy-Asobele, appendice IV.

2. Pour le sort de la négritude au Nigeria, voir Stevenson (1976), cité dans Chinweizu et al. pp. 200-204.

3. Pages 73-74 de l'original, devrait être à la page 61 de la traduction.

4. Page 170 de l'original, devrait être à la page 153 de la traduction.

5. Page 126 de l'original.

6. Page 168 de l'original, devrait être à la page 146 de la traduction.

7. Voir Antia (1988) pour d'autres exemples d'omission.

\section{RÉFÉRENCES}

Ant ia, Bassey (1988) : L'évaluation de la traduction anglaise de Le devoir de violence, Mémoire de maîtrise, Université de Lagos.

Chinweizu et al. (1976): Towards the Decolonization of African Literature, vol. I, Enugu, FDP.

King, Adele (19? ?): The Critical Reception,

Kir kup, James (trad.) (1959): The African Child, Glasgow, Fontana.

Lamb, David (1985): The Africans, New York, Vin- 